\title{
Meningitis, meningoencephalitis and encephalitis in Bern: an observational study of 258 patients
}

Anamaria Ungureanu', Julia van der Meer ${ }^{1}$, Antonela Bicvic ${ }^{1}$, Lena Abbuehl' , Gabriele Chiffi², Léonore Jaques ${ }^{1}$, Franziska Suter-Riniker ${ }^{2}$, Stephen L. Leib ${ }^{2}$, Claudio L. A. Bassetti ${ }^{1}$ and Anelia Dietmann ${ }^{1 *}$ (D)

\begin{abstract}
Background: Depending on geographic location, causes of encephalitis, meningoencephalitis and meningitis vary substantially. We aimed to identify the most frequent causes, clinical presentation and long-term outcome of encephalitis, meningoencephalitis and meningitis cases treated in the Inselspital University Hospital Bern, Switzerland.
\end{abstract}

Methods: In this monocentric, observational study, we performed a retrospective review of clinical patient records for all patients treated within a 3-year period. Patients were contacted for a telephone follow-up interview and to fill out questionnaires, especially related to disturbances of sleep and wakefulness.

Results: We included 258 patients with the following conditions: encephalitis (18\%), nonbacterial meningoencephalitis (42\%), nonbacterial meningitis (27\%) and bacterial meningoencephalitis/meningitis (13\%). Herpes simplex virus (HSV) was the most common cause of encephalitis (18\%); tick-borne encephalitis virus (TBEV) was the most common cause of nonbacterial meningoencephalitis (46\%), enterovirus was the most common cause of nonbacterial meningitis (21\%) and Streptococcus pneumoniae was the most common cause of bacterial meningoencephalitis/meningitis (49\%). Overall, 35\% patients remained without a known cause. After a median time of 16 months, 162 patients participated in the follow-up interview; 56\% reported suffering from neurological long-term sequelae such as fatigue and/or excessive daytime sleepiness (34\%), cognitive impairment and memory deficits (22\%), headache (14\%) and epileptic seizures (11\%).

Conclusions: In the Bern region, Switzerland, TBEV was the overall most frequently detected infectious cause, with a clinical manifestation of meningoencephalitis in the majority of cases. Long-term neurological sequelae, most importantly cognitive impairment, fatigue and headache, were frequently self-reported not only in encephalitis and meningoencephalitis survivors but also in viral meningitis survivors up to 40 months after acute infection.

Keywords: Encephalitis, Meningoencephalitis, Meningitis, Sleep-wake disturbances, Tick-borne encephalitis

*Correspondence: anelia.dietmann@gmail.com

${ }^{1}$ Department of Neurology, University Hopsital and University of Bern, Inselspital, Bern, Switzerland

Full list of author information is available at the end of the article

\section{Background}

Encephalitis/meningoencephalitis is an inflammation of the brain parenchyma with or without involvement of the meningeal structures. Meningitis is either a severe acute bacterial infection or less fulminant of viral origin $[1,2]$. Encephalitis is a serious and sometimes life-threatening disease that is often associated with long-term morbidity [3-5]. Considering significant geographic variation, 
encephalitis has a worldwide incidence between 1 and 13 cases/100.000/year [6, 7]. In Switzerland, tick-borne encephalitis virus (TBEV) is one of the most frequent causes of meningoencephalitis [8], whereas in the United Kingdom, herpes simplex virus (HSV) is the most common infectious cause of encephalitis [3]. Other cases of encephalitis are caused by infectious agents, including varicella zoster virus (VZV) and Mycobacterium tuberculosis, or by cellular or humoral autoimmune processes [3]. In $37-67 \%$ of patients, the cause of encephalitis remains unknown $[3-5,9]$. Similarly, in a large observational cohort study from the United Kingdom of nonbacterial meningitis cases, $42 \%$ remained without known cause, whereas enterovirus was the most common pathogen [1].

Infectious encephalitis and meningoencephalitis are associated with a high incidence of severe and debilitating long-term sequelae, whereas outcomes after autoimmune encephalitis are variable [10-14]. In contrast, viral meningitis is considered a benign, self-limiting illness; however, increasing evidence suggests that this may often not be the case [1]. In particular, signs and symptoms such as fatigue, excessive daytime sleepiness (EDS) or disturbed nighttime sleep/insomnia are frequently reported in routine clinical follow-up consultations. Fatigue as a long-term sequelae, without a detailed definition, has been described in the literature $[15,16]$ However, studies evaluating these important clinical issues have been published only recently $[17,18]$.

The aims of our study were to (1) determine the most common causes of encephalitis, meningoencephalitis and meningitis in our hospital and to (2) investigate the frequency of long-term sequelae with a focus on disorders of sleep and wakefulness (SWD).

\section{Methods}

The study was designed as a monocentric, observational study and contained two parts. The first part comprised a retrospective analysis of medical records from all patients diagnosed with any acute encephalitis, meningoencephalitis or meningitis treated in the Inselspital in Bern, Switzerland, a tertiary care university hospital with a population base of 1.5 million inhabitants. Ethical approval was given by the local Ethics Committee (Kantonale Ethikkommission Bern ID 2018-01523). Research governance approval was given at the University Hospital Inselspital, Bern, Switzerland. Medical records were only used if written general consent for research projects was available or if patients gave oral and written informed consent in the course of the telephone interview. In the second part, patients were contacted for a telephone follow-up interview and were asked to fill out and return questionnaires by mail. Oral informed consent was given during telephone interviews, and written informed consent was returned by mail.

\section{Study database}

Study data were collected and managed using REDCap electronic data capture tools hosted at the Department of Neurology, University Hospital and University of Bern, Inselspital, Bern, Switzerland [19, 20]. REDCap (Research Electronic Data Capture) is a secure, web-based software platform designed to support data capture for research studies, providing 1) an intuitive interface for validated data capture; 2) audit trails for tracking data manipulation and export procedures; 3) automated export procedures for seamless data downloads to common statistical packages; and 4) procedures for data integration and interoperability with external sources.

\section{Participants and study procedures}

Possible and consecutive participants were retrospectively identified from 1.1.2016 until 31.10 .2018 by screening the medical record database of the Inselspital University Hospital for ICD-10 Codes A83, A84, A85, B00.4, B01.0, B02.0, B05.0, B26.2, B58.2, G04, and G05 referring to all possible causes of encephalitis, meningoencephalitis or meningitis. Patients were either treated at the Department of Internal Medicine, Department of Neurology and/or the Intensive Care Unit.

Patients were eligible if aged 16 and older and had a diagnosis of encephalitis, meningoencephalitis or meningitis in their medical record, confirmed by lumbar puncture with signs of acute inflammation and/or appropriate pathogen identified either on cerebrospinal fluid (CSF) or blood PCR, serology or culture. The exclusion criteria were primary central nervous system (CNS) vasculitis, cerebral venous thrombosis, brain or spinal cord abscess, active CNS tumour or CNS lymphoma, toxic or metabolic encephalopathy or spongiform encephalopathy.

Diagnosis was reviewed and, if necessary, revised by two neurologists (A.D. and A.U.) according to published definitions [1, 21-23]. In detail, patients with acute onset of headache, fever and/or meningism, CSF leucocyte count $>4 \times 10^{6}$ cells $/ \mathrm{L}$ and, if possible, detection of an appropriate pathogen by either CSF PCR, blood/CSF serology or blood/CSF culture or throat or rectal swab were classified as having meningitis [1]. Meningoencephalitis was classified when signs of meningitis were present plus altered consciousness and/or focal neurological symptoms and/or abnormal findings in EEG [10]. Encephalitis was defined as altered consciousness for $>24 \mathrm{~h}$ with no other cause and evidence of CNS inflammation, demonstrated by at least two of the following criteria: fever, seizures or focal neurological findings attributable to the brain parenchyma, CSF 
pleocytosis (CSF leucocyte count $>4 \times 10^{6}$ cells per L), EEG findings suggestive of encephalitis and/or neuroimaging findings suggestive of encephalitis [21]. For $\mathrm{TBE}$, the following case definition was applied: patients with symptoms of CNS inflammation (meningitis, meningoencephalitis or encephalitis criteria), history of possible exposure or tick bites and detection of TBEspecific IgM and IgG antibodies in serum using the SERION ELISA classic FSME virus/TBE virus IgG and IgM kit (Virion\Serion, Würzburg, Germany). In cases where only IgM antibodies are detected, a follow-up sample is needed to demonstrate IgG seroconversion and thereby establish the diagnosis [22, 23].

Detection of TBE viral nucleic acid in blood by PCR or isolation of TBE virus was not performed.

The FilmArray ${ }^{\circledR}$ ME Panel (BioFire, bioMerieux, Salt Lake City, USA) was used. Two hundred microlitres of CSF was subjected to FilmArray ${ }^{\circledR}$ ME Panel testing according to the manufacturer's instructions. The FilmArray ${ }^{\circledR}$ ME Panel test consists of automated sample homogenization and nucleic acid extraction, reverse transcription, and nucleic acid amplification. The FilmArray ${ }^{\circledR}$ ME Panel identifies 14 common agents of community-acquired ME: Escherichia coli K1, Haemophilus influenzae, Listeria monocytogenes, Neisseria meningitidis, Streptococcus pneumoniae, Streptococcus agalactiae, cytomegalovirus (CMV), enterovirus (EV), herpes simplex virus type 1 (HSV-1), herpes simplex virus type 2 (HSV-2), human herpesvirus type 6 (HHV6), human parechovirus $(\mathrm{HPeV})$, varicella zoster virus (VZV), and Cryptococcus neoformans/gattii from CSF.

Bacterial meningitis cases were diagnosed by culture.

Borrelia Burgdorferi: serological testing of IgG and IgM using IgG and IgM recomWell ELISA (recomWell, Mikrogen) according to the manufacturer's instructions. In the case of positive screening test results, the $\mathrm{CSF} /$ serum antibody index was determined using IgG and IgM ELISA Virion/Serion (Würzburg, Germany) according to the manufacturer's instructions. In this case, the presence of Borrelia-specific antibodies in simultaneously sampled serum and CSF probes was determined.

Treponema pallidum infection: reactive VRDL in CSF and/or a positive CSF intrathecal $\mathrm{T}$ pallidum antibody index.

Preexisting diseases, immune suppressive state and signs and symptoms pre- and in the hospital were taken from the medical records. Furthermore, findings in the neurological examinations, laboratory findings and microbiological results were taken from the clinical record. At hospital discharge, a modified Rankin score (mRS) was taken from the clinical record if available or calculated as described in the clinical record.

\section{Follow-up}

Clinical outcomes and subjective long-term sequelae were assessed with a standardized interview via telephone (A.U., L.J.) from October 2019 until February 2020. Patients were contacted twice, first for information and informed consent procedures and a second time for the interview. Following questionnaires were sent out immediately after the telephone interview to the study participants evaluating sleep-wake-disorders including Epworth Sleepiness Scale (ESS, self-administered evaluation of daytime sleepiness )[24], Fatigue Severity Scale (FSS, self-administered evaluation of fatigue, designed to differentiate fatigue from clinical depression )[25], Insomnia Severity Index (ISI, self-administered evaluation of severity of both nighttime and daytime components of insomnia )[26], Beck Depression Inventory II (BDI II, self-administered evaluation of severity of depression )[27]. Cut-offs for pathological scores were set according to the literature: ESS $>9$, FSS $>4$, ISI $>7$, BDI II $>8$. All questionnaires are frequently used in clinical routine as well as for scientific purposes and have been frequently used in or validated for Swiss patients [25, 28-31]. Furthermore, a selected set of individual questions regarding SWD from the Bern Sleep Questionnaire were sent to the study participants (see supplementary data). The full questionnaire used for clinical routine in our sleep centre has been described elsewhere [31]. Four questions regarding restless leg syndrome (RLS) were included according to diagnostic consensus criteria [32].

\section{Statistical analysis}

Nonparametric continuous data were analysed by using Kruskal-Wallis tests. Categorical data were analysed by using $X^{2}$ test. Correlation coefficients ( $R$ values) were obtained using Pearson's correlation analysis. A $p$ value of less than 0.05 was considered statistically significant. Missing data were not imputed. We statistically analysed data using Stata/MP 16.0 and R 3.6.1, and graphs were drawn by GraphPad Prism 8, Stata/MP 16.0 and R 3.6.1.

\section{Results}

From a total of 463 screened patient medical records, 258 patients were included in the retrospective analysis of the study (Fig. 1).

\section{Distribution of diagnoses and causes}

In total, as shown in Table 1, during the 34-month period, 258 patients were treated for encephalitis (46, $18 \%)$, nonbacterial meningoencephalitis (109, 42\%), nonbacterial meningitis $(70,27 \%)$ or bacterial meningoencephalitis/meningitis (33, 13\%).

As shown in Table 2, the most frequent causes overall were of infectious origin (57\%); in 5\%, an 


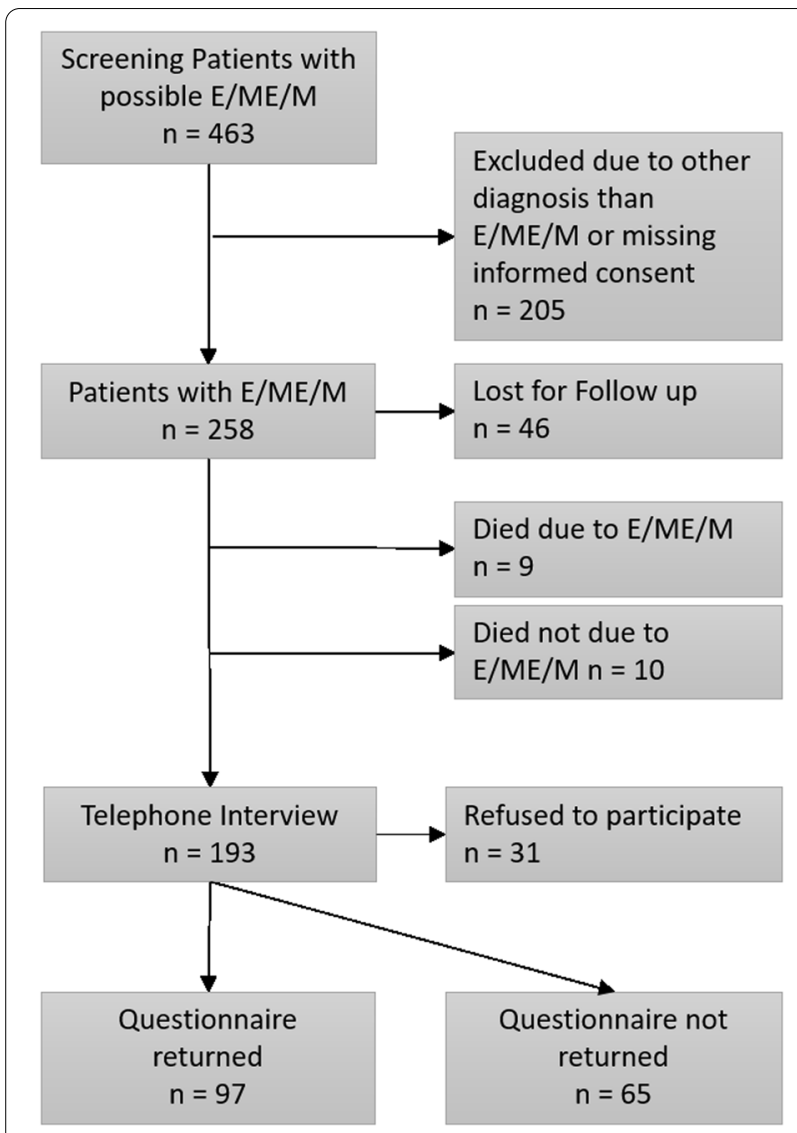

Fig. 1 Flow chart of patient recruitment and data acquisition. Legend: E/ME/M encephalitis, meningoencephalitis or meningitis

immune-mediated cause was found, and 36\% remained without a known cause.

TBEV was the most frequently detected cause of meningoencephalitis or meningitis (65/258 cases, $25 \%)$ in our overall patient population; $58(89 \%)$ presented with meningoencephalitis (including 9 cases of meningoencephalomyelitis and meningoencephalomyeloradiculitis), $6(9 \%)$ with meningitis and $1(2 \%)$ with encephalitis. In the meningoencephalitis group, 6 patients were classified as having meningoencephalomyelitis (causes: $2 \mathrm{VZV}$ and $4 \mathrm{TBE}$ ), and 7 were classified as having meningoencephalomyeloradiculitis (causes: 5 TBE, 1 unknown and 1 suspected parainfectious cause with Bartonella henselae infection). In the encephalitis group, in 13/46 (28\%) patients, an autoimmune cause was found. Recurrent meningitis was seen in 4 patients: one had 2 episodes within 6 months and was later suspected to have systemic lupus erythaematodes, one had 3 episodes (2007 and 2 episodes in 2017) without known cause, one had 2 episodes of enteroviral meningitis within 3 years and one had 2 episodes of self-limiting treatment-induced meningitis within 3 months during treatment of myelodysplastic syndrome with antibiotics, virostatic and corticosteroid treatment.

\section{Clinical presentation}

As shown in detail in Table 1, the median time from the onset of signs and symptoms until hospital admission was 5.5 days in encephalitis, 7 days in nonbacterial meningoencephalitis, 4days in nonbacterial meningitis and 2 days in bacterial meningoencephalitis/meningitis.

Three patients presented with meningitis within $48 \mathrm{~h}$ after IVIG infusion with the main symptoms of headache, phono- and/or photophobia and nausea and had mild pleocytosis with normal protein values. All patients had a mRS of 0 at hospital discharge.

\section{Follow-up}

The telephone follow-up interview took place at a median of 16 months (range 2-40 months) after hospital discharge (Supplementary Table 1) in 193 patients. Forty-six patients were lost to follow-up, and 19 died; 9 died due to acute encephalitis or meningoencephalitis. All interviewed patients were living at home, and the majority (96-100\%) were able to cook their own meal, perform the laundry, and use public transport unaided independently of the diagnostic group. However, only $50 \%$ of encephalitis, $75 \%$ of bacterial meningoencephalitis/meningitis, $81 \%$ of nonbacterial meningoencephalitis and $94 \%$ of nonbacterial meningitis survivors were able to restart work or studies to the same extent as before the acute illness. Overall, 37\% of patients indicated not feeling completely fit again (encephalitis 65\%, nonbacterial meningoencephalitis $39 \%$, nonbacterial meningitis $16 \%$, bacterial meningoencephalitis/meningitis 53\%). When asked whether to still feel more rapidly exhausted physically or mentally compared to before the acute illness, $47 \%$ of patients agreed (encephalitis $70 \%$, nonbacterial meningoencephalitis $45 \%$, nonbacterial meningitis $32 \%$, bacterial meningoencephalitis/meningitis $71 \%$ ).

Persisting neurological manifestations were reported by $56 \%$ of patients irrespective of elapsed time since acute illness (encephalitis 83\%, nonbacterial meningoencephalitis $54 \%$, nonbacterial meningitis $42 \%$, bacterial meningoencephalitis/meningitis $71 \%$, Supplementary Table 1). The most frequent manifestations were EDS and/or fatigue (34, 95\% CI 27-41, Supplementary Fig. 1), cognitive impairment (22\%; 29-16), headache (14\%; 9-20) and epileptic seizures (11\%; 6-16). Regarding headache, 9\% (4-13) indicated suffering from headache less than 15 days per month, and 6\% (2-9) reported suffering more than 15 days per month. 
Table 1 Demographics, clinical signs and symptoms and summary of cerebrospinal fluid investigations of all study patients and comparison between groups of encephalitis, nonbacterial meningoencephalitis, nonbacterial meningitis and bacterial meningoencephalitis or meningitis defined by the clinical syndrome

\begin{tabular}{|c|c|c|c|c|c|c|}
\hline & All & Encephalitis & $\begin{array}{l}\text { Nonbacterial } \\
\text { Meningo- } \\
\text { encephalitis }\end{array}$ & $\begin{array}{l}\text { Nonbacterial } \\
\text { Meningitis }\end{array}$ & $\begin{array}{l}\text { Bacterial } \\
\text { Meningoencephalitis } \\
\text { or Meningitis }\end{array}$ & $p$ value $^{b}$ \\
\hline Total n (\%) & $258(100)$ & $46(17.8)$ & $109(42.2)$ & $70(27.1)$ & $33(12.8)$ & \\
\hline Sex female $n(\%)$ & $126(48.8)$ & $18(39.1)$ & $53(48.6)$ & $41(58.6)$ & $14(42.4)$ & 0.18 \\
\hline Median age (IQR) & $51.5(33)$ & $60(24)$ & $56(36)$ & $38(28)$ & $53(19)$ & $<0.0001$ \\
\hline $\begin{array}{l}\text { Median days from clinical onset to hospital } \\
\text { admission (IQR) }\end{array}$ & $5(8)$ & $5.5(18)$ & $7(10)$ & $4(5)$ & $2(5)$ & $<0.0003$ \\
\hline \multicolumn{7}{|l|}{ Symptoms or clinical signs } \\
\hline Headache $e^{a}$ & $183(71,65-76)$ & $12(26,15-41)$ & $85(78,69-85)$ & $63(90,80-95)$ & $23(70,52-83)$ & \\
\hline Fever & $150(58,52-64)$ & $17(37,24-52)$ & $73(67,58-75)$ & $38(54,42-66)$ & $22(67,49-81)$ & \\
\hline Meningism & $77(30,25-36)$ & $2(4,1-16)$ & $29(27,19-36)$ & $27(39,28-50)$ & $19(58,40-73)$ & \\
\hline Photo-/Phonophobia & $47(18,14-23)$ & $0(0)$ & $16(15,9-23)$ & $27(39,9-23)$ & $4(12,5-28)$ & \\
\hline Nausea/vomiting & $98(38,32-44)$ & $8(17,9-31)$ & $40(37,28-46)$ & $40(57,45-68)$ & $10(30,17-48)$ & \\
\hline Epileptic Seizures & $73(29,23-34)$ & $27(59,44-72)$ & $32(29,22-39)$ & $2(2,1-11)$ & $12(36,22-54)$ & \\
\hline Sensory/motor deficits & $66(26,21-31)$ & $12(26,15-41)$ & $38(35,27-44)$ & $7(10,5-20)$ & $9(27,15-45)$ & \\
\hline Confusion & $74(29,24-35)$ & $27(59,44-72)$ & $31(28,21-38)$ & $6(9,4-18)$ & $10(30,17-48)$ & \\
\hline Cognitive impairment & $70(27,22-33)$ & $24(52,38-66)$ & $36(33,25-42)$ & $4(6,2-14)$ & $6(18,8-35)$ & \\
\hline Abnormal behaviour & $53(21,16-26)$ & $23(50,36-64)$ & $23(21,14-30)$ & $3(4,1-13)$ & $4(12,5-28)$ & \\
\hline Aphasia & $55(21,17-27)$ & $16(35,23-50)$ & $35(32,24-42)$ & $0(0)$ & $4(12,5-28)$ & \\
\hline Vertigo & $48(19,14-24)$ & $9(19,11-34)$ & $28(26,18-35)$ & $9(13,7-23)$ & $2(6,2-21)$ & \\
\hline Gait disturbance & $45(17,13-23)$ & $11(24,14-38)$ & $28(26,18-35)$ & $2(3,1-11)$ & $4(12,5-28)$ & \\
\hline Cerebellar signs & $36(14,10-19)$ & $10(22,12-36)$ & $24(22,15-31)$ & $1(1,0-10)$ & $1(3,1-19)$ & \\
\hline Cranial nerve dysfunction & $37(14,11-19)$ & $2(4,1-16)$ & $24(22,15-31)$ & $4(6,2-14)$ & $7(21,10-38)$ & \\
\hline Impaired consciousness & $101(39,33-45)$ & $27(59,44-72)$ & $46(42,33-52)$ & $2(3,1-11)$ & $26(79,62-90)$ & \\
\hline $\mathrm{GCS}<15$ & $91(35,30-41)$ & $23(54,40-68)$ & $34(37,28-46)$ & $1(0-10)$ & $24(76,58-87)$ & \\
\hline GCS median (IQR) & $9(6)$ & $8(9)$ & $10(6)$ & $13(0)$ & $9(3)$ & \\
\hline Mechanical ventilation & $45(17,13-23)$ & $12(26,15-41)$ & $17(16,10-24)$ & $0(0)$ & $16(49,32-65)$ & \\
\hline mRS (IQR) & $2(2)$ & $3(2)$ & $2(2)$ & $1(1)$ & $3(1)$ & \\
\hline \multicolumn{7}{|l|}{ CSF results } \\
\hline Median leucocyte count ( $\times 10^{6}$ per L, IQR) & $81.5(190.5)$ & $14(55)$ & $75.5(148)$ & $96(194)$ & $1218.5(5946)$ & $<0.0001$ \\
\hline $\begin{array}{l}\text { Proportion lymphocytes of white blood } \\
\text { cells }(\%, I Q R)\end{array}$ & $92(49.5)$ & $99(7)$ & $94(32)$ & $91.5(25)$ & $10(23)$ & $<0.0001$ \\
\hline Median protein (g/L, IQR) & $0.78(0.61)$ & $0.66(0.54)$ & $0.79(0.43)$ & $0.58(0.41)$ & $3.36(6.63)$ & $<0.0001$ \\
\hline Median lactate (mmol/L, IQR) & $2.5(1.09)$ & $2.2(0.8)$ & $2.5(0.75)$ & $2.3(1)$ & $13(11.9)$ & $<0.0001$ \\
\hline
\end{tabular}

Legend: ${ }^{\mathrm{D} D a t a}$ are the number of patients with each sign or symptom on admission and/or course of acute disease (\%, 95 confidence interval Cl), IQR interquartile range $75-25$, bruskal-Wallis equality-of-populations rank test comparing all 4 diagnostic groups. Categorical data were analysed by $x^{2}$ test comparing all 4 diagnostic groups. CSF cerebrospinal fluid, GCS Glasgow Coma Scale, mRS modified Rankin Scale

\section{Follow-up questionnaires}

Overall, 97 patients returned questionnaires (Fig. 2), including the Epworth Sleepiness Score (ESS), Fatigue Severity Score (FSS), Insomnia Severity Index (ISI) and Beck Depression Index II (BDI II). Overall, the proportions of pathological scores were 23\% (95\% CI 15-32) for ESS (cut-off >10), 24\% (16-33) for FSS (cut-off $>4), 40 \%(31-50)$ for ISI (cut-off > 7) and 31\% (23-41) for BDI II (cut-off $>8$ ). The proportion of pathological scores was not significantly different between groups (ESS $p=0.258$, FSS $p=0.06$ ISI $p=0.403$, BDI II $p=0.077$ ). Time since hospital discharge did not significantly influence any score value (ESS R $=0.0002,95 \%$ CI $-0.86-0.1, p=0.885$; FSS $\mathrm{R}=0$, 95\% CI $-0.04-0.04$, $p=0.967$; ISI $\mathrm{R}=0.012,95 \% \mathrm{CI}-0.206-0.061, p=0.285$; BDI II $\mathrm{R}=0.0001,95 \%$ CI $-0.148-0.16, p=0.08)$.

In a set of questions regarding sleep-wake disorders, 39 and $42 \%$ (95\% CI 30-49 and 33-52, respectively) indicated suffering from excessive daytime sleepiness 
Table 2 Confirmed causes of encephalitis, meningoencephalitis or meningitis

\begin{tabular}{|c|c|c|c|c|}
\hline Total $n(\%)$ & $\begin{array}{l}\text { All } \\
258(100)\end{array}$ & $\begin{array}{l}\text { Encephalitis } \\
46(17.8)\end{array}$ & $\begin{array}{l}\text { Meningoencephalitis } \\
127(49.2)\end{array}$ & $\begin{array}{l}\text { Meningitis } \\
85 \text { (32.9) }\end{array}$ \\
\hline Infectious cause & $148(57.4)$ & $15(32.6)$ & $89(70.1)$ & $45(52.9)$ \\
\hline Viral & $114(77)$ & $14(93.3)$ & $71(79.8)$ & $30(68.2)$ \\
\hline Tick-borne Encephalitis Virus & $65(57)$ & $1(7.1)$ & $58(81.7)$ & $6(20.7)$ \\
\hline Enterovirus & $21(18.4)$ & $1(7.1)$ & $3(4.2)$ & $18(60)$ \\
\hline Varicella zoster virus & $14(12.3)$ & $2(14.2)$ & $7(9.9)$ & $5(17.2)$ \\
\hline Herpes Simplex virus 1 & $9(7.9)$ & $8(57.1)$ & $1(1.4)$ & $0(0)$ \\
\hline Herpes Simplex virus 2 & $2(1.8)$ & $0(0)$ & $1(1.4)$ & $1(3.4)$ \\
\hline Epstein-Barr-Virus & $2(1.8)$ & $1(7.1)$ & $1(1.4)$ & $0(0)$ \\
\hline Influenza virus A/B & $1(0.9)$ & $1(7.1)$ & $0(0)$ & $0(0)$ \\
\hline Bacterial & $34(23)$ & $1(6.7)$ & $18(20.2)$ & $15(34.1)$ \\
\hline Streptococcus pneumoniae & $16(48.5)$ & & $9(50)$ & $7(46.7)$ \\
\hline Neisseria meningitidis & $4(12.1)$ & & $3(16.7)$ & $1(6.7)$ \\
\hline Streptococcus ssp. & $4(12.1)$ & & $2(11.1)$ & $2(13.3)$ \\
\hline Haemophilus influenzae & $2(6.1)$ & & $1(5.6)$ & $1(6.7)$ \\
\hline Listeria monocytogenes & $1(3)$ & & $0(0)$ & $1(6.7)$ \\
\hline Staphylococcus aureus & $1(3)$ & & $0(0)$ & $1(6.7)$ \\
\hline Tuberculosis & $3(9.1)$ & & $2(11.1)$ & $1(6.7)$ \\
\hline Borrelia burgdorferi & $2(6.1)$ & & $1(5.6)$ & $1(6.7)$ \\
\hline Treponema pallidum & $1(0.7)$ & $1(6.7)$ & $0(0)$ & $0(0)$ \\
\hline Immune-mediated cause & $13(5)$ & $13(28.3)$ & $0(0)$ & $0(0)$ \\
\hline NMDA-Receptor-Antibody & $4(30.7)$ & $4(30.7)$ & & \\
\hline LGl1 Antibody & $4(30.7)$ & $4(30.7)$ & & \\
\hline Caspr2 & $2(15.4)$ & $2(15.4)$ & & \\
\hline Anti-Hu & $1(7.7)$ & $1(7.7)$ & & \\
\hline GAD & $1(7.7)$ & $1(7.7)$ & & \\
\hline SREAT & $1(7.7)$ & $1(7.7)$ & & \\
\hline Other & $5(1.9)$ & $0(0)$ & $0(0)$ & $5(5.9)$ \\
\hline Intravenous Immunoglobuline & $3(60)$ & & & $3(60)$ \\
\hline Craniopharyngioma & $1(20)$ & & & $1(20)$ \\
\hline Autoimmune Disease & $1(20)$ & & & $1(20)$ \\
\hline Unknown cause & $92(35.7)$ & $18(39.1)$ & $38(29.9)$ & $35(41.2)$ \\
\hline
\end{tabular}

Legend: Data are the number of patients with each clinically defined syndrome (\%). Streptococcus ssp.: pyogenes, viridans, milleri, agalactiae; NMDA methyl D-aspartate receptor, LGI-1 leucine-rich, glioma inactivated 1, Caspr2 contact associated protein 2, GAD glutamic acid decarboxylase, SREAT steroid-responsive encephalopathy with autoimmune thyroiditis

(agreed to questions: "I am tired during the day and I have to fight to stay awake and against sleeping in" and "It happens frequently, that I am forced to take a nap."). However, 54\% (95\% CI 44-63) also indicated suffering from fatigue (agreed to question: "During the Day I feel exhausted and tired, however I am not able to sleep in when given a possibility to nap."). New onset of daytime SWD, such as fatigue and EDS since suffering from the acute illness, was indicated by $25 \%$ (95\% CI $17-34$ ); $33 \%$ (24-43) thereof indicated having been suffering from the same problems already before the acute illness, and $42 \%$ (33-52) could not indicate clear onset of fatigue or EDS.
Overall, 22 patients out of 97 answered all 4 diagnostic RLS questions positively and accordingly fulfilled the diagnostic criteria of RLS. Twelve patients indicated having experienced new onset of RLS since the acute illness.

Five out of 97 patients indicated new onset of hypnagogic or hypnopompic hallucinations since the acute illness. No patient reported new onset of episodes indicating REM-sleep behaviour disorder, sleep paralysis or cataplexy since surviving the acute illness. 


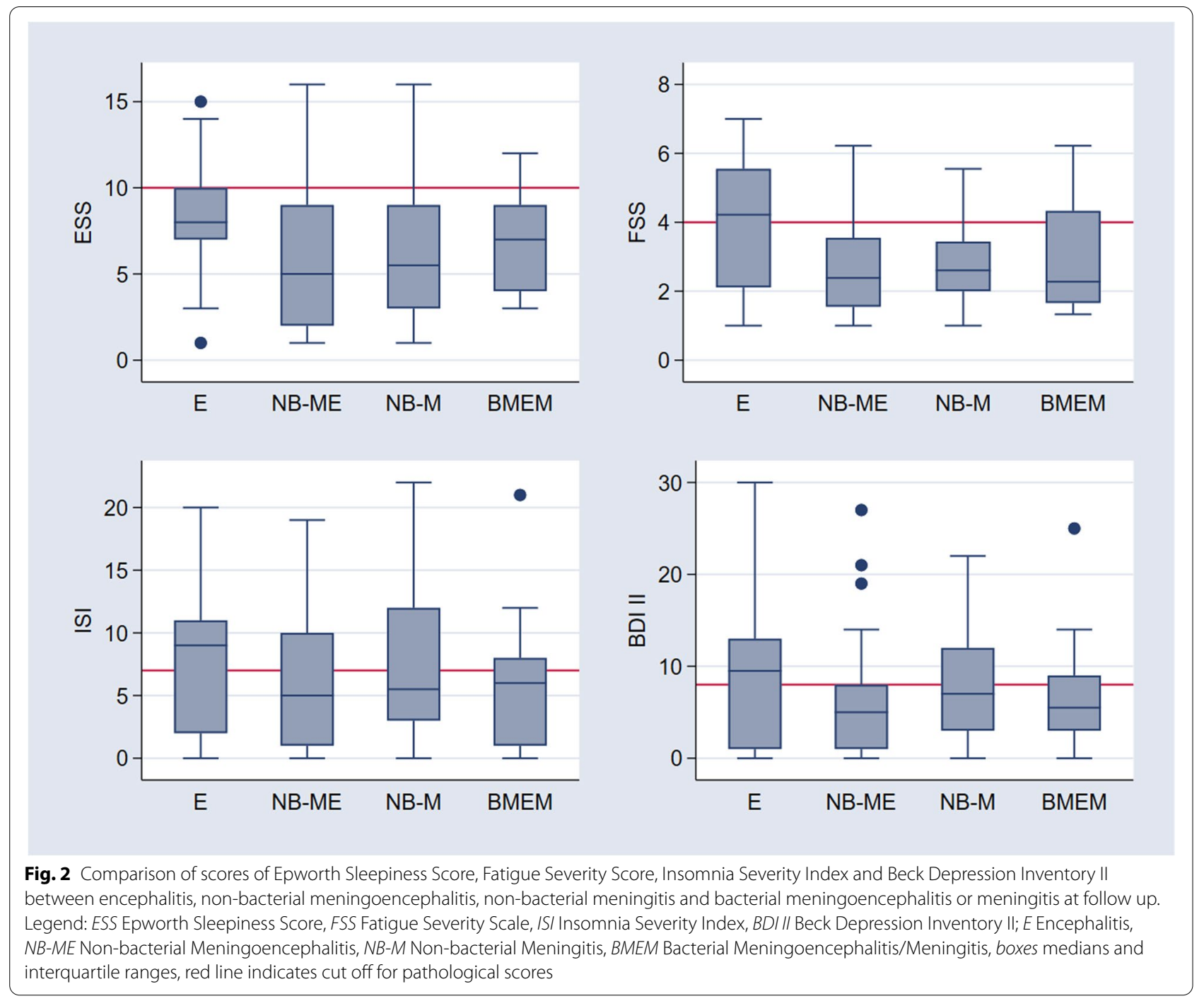

\section{Discussion}

There were three main findings in this study. First, in our hospital in Switzerland, TBEV is the most important overall infectious cause of encephalitis, meningoencephalitis and meningitis, presenting as meningoencephalitis in the majority of patients. Second, only one-third of patients remain without a detectable cause of acute illness. Third, neurologic sequelae, most importantly cognitive impairment, fatigue and headache, were selfreported in a significant proportion not only of encephalitis, meningoencephalitis and bacterial meningitis survivors but also of nonbacterial meningitis patients up to 40 months after surviving the acute illness.

HSV was the most common cause of encephalitis, followed by immune-mediated causes. Therefore, together with VZV encephalitis, almost three-quarters of our encephalitis cases had a treatable cause. Depending on geographic location, these findings are in line with other
European countries [1, 3, 33, 34]. While early detection and treatment of herpes encephalitis is essential for prevention of fatal outcome [35], recognition of autoimmune causes is of utmost importance since they belong to an expanding group of potentially treatable and curable causes of encephalitis and likely benefit from early immunosuppressive treatment regimens [36, 37]. Nonbacterial meningoencephalitis was most importantly caused by TBEV, since it is endemic in most parts of Switzerland with a nationwide estimated incidence of 6.83 cases per 100,000 unvaccinated inhabitants and nationwide vaccine coverage of only $42 \%$ for 1 dose and $33 \%$ for full vaccination with 3 doses in 2018 [38]. Since TBE vaccination is generally well tolerated with high rates of seroconversion, it has been recommended for all age groups above 1 year in highly endemic areas and for individuals at risk in areas with a lower incidence, including travellers with outdoor activities [22]. Higher vaccine coverage 
in Switzerland would most likely have an effect on local meningoencephalitis incidence; many cases could therefore be prevented.

In line with large European studies, Streptococcus pneumoniae was the main detected cause of bacterial meningitis/meningoencephalitis [2]. Finally, in line with other studies, including a large cohort study from the United Kingdom [1], enterovirus was the most important cause of nonbacterial meningitis. Interestingly, three patients were hospitalized with drug-induced meningitis due to intravenous immunoglobulin (IVIG) treatment, a rare side effect described in the literature occurring in up to $1 \%$ of cases receiving IVIG therapy [39]. Overall, $35 \%$ of cases remained without a known cause, which is in line with other studies [1,3-5]. Causative agents in this group of patients remain speculative. However, recent studies have demonstrated increased diagnostic yield using nextgeneration sequencing methods to identify further causative infectious pathogens $[40,41]$.

At follow-up - independent of elapsed time of up to 40 months after acute illness - more than one-third of patients indicated that they had not regained full fitness, and more than half complained about neurological sequelae, despite functioning well in everyday activities such as cooking, doing laundry and financial affairs. However, neurological sequelae were frequently reported, mostly in encephalitis (83\%) and bacterial meningoencephalitis/ meningitis survivors (71\%) but also in meningoencephalitis $(54 \%)$ and nonbacterial meningitis (42\%) survivors. Comparable findings have been described for encephalitis and meningoencephalitis, including TBE $[10,12,13]$. Our results are also in line with studies on viral meningitis, demonstrating long-lasting sequelae despite its ostensibly benign course [1, 42-44].

However, only half of encephalitis and $75 \%$ of bacterial meningoencephalitis/meningitis survivors were able to return to work. In a recent cohort study on communityacquired bacterial meningitis in adults, only $62 \%$ were able to return to work [45], and our higher proportion of survivors able to return to work might be due to a selection bias in our telephone interview, as discussed below.

To our knowledge, only a few studies have been published using standardized questionnaires on EDS, fatigue and insomnia in the field of encephalitis, meningoencephalitis and meningitis [17, 18]. In our study, EDS and/or fatigue were frequently reported in the follow-up; however, precise discrimination between the two clinically different symptoms was not possible. Insomnia/disturbed nighttime sleep was less frequently reported. Thirty-one percent of patients scored a pathological BDI II; however, the majority of patients had scores below 20 points, except encephalitis, with up to 30 points indicating mild to moderate depression. Since fatigue and insomnia may be key features of depression, interactions must be taken into account when using questionnaires in study settings as well as routine clinical practice. Symptoms need to be carefully evaluated to distinguish the origin of overlapping signs and symptoms.

The limitations of our study are the retrospective analysis of clinical cases in the acute phase with less precise data on clinical signs and symptoms and therefore diagnostic classification. In the retrospective use of patient data, we probably missed cases where general consent for further data use was unavailable. The telephone followup and the relatively low number of returned questionnaires might introduce bias in two possible directions.

First, it is possible that survivors with better outcomes might have been more willing to participate in the study. Since it is mostly not possible to contact severely disabled patients via telephone, this could potentially account for our number of lost-for follow-up patients. Therefore, it could be speculated that the true overall outcome of our full study population may be worse than that described in our study. Alternatively, it is possible that patients with sequelae may have been more motivated to participate in the telephone interview than those who had completely recovered. This scenario would therefore imply an overall better outcome than that reported in our study. Important value to the study and an attempt to solve these problems would have been the inclusion of a control group hospitalized for other acute illnesses treated in the hospital not affecting the CNS. Moreover, outcome data are difficult to interpret regarding the different time points when the follow-up survey took place. Taken together, our results on long-term outcomes have to be interpreted with care and need validation in further, ideally prospective studies. Important points for future studies are to include a personal follow-up visit for a detailed interview and a physical examination to better interpret and underline subjective complaints as well as evaluation of quality of life and the impact of persisting signs and symptoms on everyday life. Another important topic not addressed in our study is the importance of elapsed time from symptom onset until diagnosis and treatment or even time from "door to diagnosis and treatment" and its effect on functional long-term outcome.

Encephalitis and meningoencephalitis are often difficult to distinguish in routine clinical practice and have overlapping features and causes. This can also be seen in our encephalitis patients, whereof $26 \%$ had headache, and in 2 patients, even signs of meningism were documented. Therefore, clinical signs and symptoms may depict a continuum between meningoencephalitis and encephalitis for the diagnostic work-up at the emergency department. Nevertheless, it is important to try to classify the clinical 
syndrome to direct the diagnostic steps in the right direction of the most likely and most important causes.

\section{Conclusions}

With this observational study including 258 patients, we demonstrate the importance of TBEV as the major cause of encephalitis, meningoencephalitis and meningitis cases in our geographic region and its primary clinical presentation as meningoencephalitis. Furthermore, we were able to assess long-lasting neurological sequelae not only after encephalitis, meningoencephalitis or bacterial meningitis but also after nonbacterial meningitis in a relevant proportion of patients up to 40 months post infection.

\begin{abstract}
Abbreviations
BDI-II: Beck Depression Inventory II; Caspr2: Contactin associated protein 2; Cl: Confidence Interval; CMV: Cytomegalovirus; CNS: Central Nervous System; CSF: Cerebrospinal Fluid; EBV: Ebstein Barr Virus; EDS: Excessive Daytime Sleepiness; EEG: Electroencephalography; ESS: Epworth Sleepiness Scale; FSS: Fatigue Severity Scale; GAD: Glutamic acid decarboxylase; GCS: Glasgow Coma Scale; HSV: Herpes Simplex Virus; HHV: Human Herpes Virus; ISS: Insomnia Severity Index; IQR: Interquartile range; LGl-1: Leucine-rich, glioma inactivated 1; mRS: Modified Rankin Score; NMDA: Methyl D-aspartate receptor; PCR: Polymerase Chain Reaction; REDCap: Research Electronic Data Capture; RLS: Restless Legs Syndrome; SREAT: Steroid-responsive encephalopathy with autoimmune thyroiditis; SWD: Sleep Wake Disorders; TBE: Tick borne encephalitis; TBEV: Tick borne encephalitis virus; VZV: Varicella Zoster Virus; WNV: West Nile Virus.
\end{abstract}

\section{Supplementary Information}

The online version contains supplementary material available at https://doi. org/10.1186/s12883-021-02502-3.

Additional file 1: Supplementary Table 1. Follow up Interview. Supplementary Fig. 1. Follow-up Interview: self-reported neurological signs and symptoms persist up to 40 months after hospitalization. For each neurological symptom, individual timing of the follow-up interview relative to hospitalization is illustrated with dot plots. Individual data are summarized as Box plots.

\section{Acknowledgements}

Anelia Dietmann has been personally supported by research grants from the "Bangerter Rhyner Stiftung" and a CTU Research Grant of the University of Bern. Gabriele Chiffi has been supported by an Interfaculty Research Cooperation Grant "Decoding Sleep" by the University of Bern, Switzerland.

We thank the ENCEIF Study Group and the European Society of Clinical Microbiology and Infectious Diseases Study Group on Infection of the Brain (ESGIB) for important input on study design and follow-up interviews.

\section{Authors' contributions}

AU: Drafting/revision of the manuscript for content, including medical writing for content; Major role in the acquisition of data; Analysis and interpretation of data. JVDM: Revision of the manuscript for content, including medical writing for content; Analysis and interpretation of data. AB: Revision of the manuscript for content, including medical writing for content; Major role in the acquisition of data. LA: Revision of the manuscript for content; Major role in the acquisition of data. LJ: Revision of the manuscript for content; Major role in the acquisition of data. GC: Revision of the manuscript for content; Analysis of data. FSR: Revision of the manuscript for content, including medical writing for content; Major role in the acquisition of data; Study concept. SLL: Revision of the manuscript for content, including medical writing for content; Study concept and design; Analysis or interpretation of data. CLAB: Revision of the manuscript for content, including medical writing for content; Study concept or design; Interpretation of data. AD: Drafting and revision of the manuscript for content, including medical writing for content; Major role in the acquisition of data; Study concept and design; Analysis and interpretation of data. The author(s) read and approved the final manuscript.

\section{Funding}

Anelia Dietmann has been personally supported by research grants from the "Bangerter Rhyhner Stiftung" and a CTU Research Grant of the University of Bern. Both funding bodies had no influence on study design, collection, analysis and interpretation of data or writing the manuscript. Gabriele Chiffi has been supported by an Interfaculty Research Cooperation Grant "Decoding Sleep" by the University of Bern, Switzerland. The funding body had no influence on study design, collection, analysis and interpretation of data or writing the manuscript.

\section{Availability of data and materials}

Anonymized data will be shared with qualified investigators upon contacting the corresponding author.

\section{Declarations}

\section{Competing of interests}

On behalf of all authors, the corresponding author states that there are no competing of interests.

\section{Ethics approval and consent to participate}

The study was approved by the Kantonale Ethikkommission Bern ID 201801523. Research governance approval was given at the University Hospital Inselspital, Bern, Switzerland. Retrospective data were used if a written general consent (written declaration of consent for the use of health-related data and samples for research purposes) had been signed and was available in the patients' medical records. All participants of the prospective part of the study gave written informed consent or agreed to general consent.

\section{Consent for publication}

Not applicable.

\section{Author details}

${ }^{1}$ Department of Neurology, University Hopsital and University of Bern, Inselspital, Bern, Switzerland. ${ }^{2}$ Institute for Infectious Disease, University of Bern, Bern, Switzerland.

Received: 19 April 2021 Accepted: 25 November 2021

Published online: 06 December 2021

\section{References}

1. Mcgill F, Griffiths MJ, Bonnett LJ, Geretti AM, Michael BD, Beeching NJ, et al. Incidence, aetiology, and sequelae of viral meningitis in UK adults: a multicentre prospective observational cohort study. Lancet Infect Dis. 2018;18:992-1003. https://doi.org/10.1016/S1473-3099(18)30245-7 www.thelancet.com/infection.

2. van de Beek D, Cabellos C, Dzupova O, Esposito S, Klein M, Kloek AT, et al. ESCMID guideline: diagnosis and treatment of acute bacterial meningitis. Clin Microbiol Infect. 2016;22:S37-62. https://doi.org/10. 1016/j.cmi.2016.01.007.

3. Granerod J, Ambrose HE, Davies NW, Clewley JP, Walsh AL, Morgan D, et al. Causes of encephalitis and differences in their clinical presentations in England: a multicentre, population-based prospective study. Lancet Infect Dis. 2010;10:835-44. https://doi.org/10.1016/S14733099(10)70222-X.

4. Glaser CA, Gilliam S, Schnurr D, Forghani B, Honarmand S, Khetsuriani $N$, et al. In search of encephalitis etiologies: diagnostic challenges in the California encephalitis project, 1998-2000. Clin Infect Dis. 2003;36:731-42. https://doi.org/10.1086/367841.

5. Mailles A, Stahl J. Steering committee and investigators group. Infectious encephalitis in France in 2007: a National Prospective Study. Clin Infect Dis. 2009;49:1838-47. https://doi.org/10.1086/648419. 
6. Granerod J, Tam CC, Crowcroft NS, Davies NWS, Borchert M, Thomas SL. Challenge of the unknown: a systematic review of acute encephalitis in non-outbreak situations. Neurology. 2010;75:924-32. https://doi.org/10. 1212/WNL.0b013e3181f11d65.

7. Boucher A, Herrmann JL, Morand P, Buzelé R, Crabol Y, Stahl JP, et al. Epidemiology of infectious encephalitis causes in 2016. Med Mal Infect. 2017;47:221-35.

8. Altpeter E, Zimmermann H, Oberreich J, Péter O, Dvorák C, Swiss S. Tick related diseases in Switzerland, 2008 to 2011. Swiss Med Wkly. 2013;143:w13725. https://doi.org/10.4414/smw.2013.13725.

9. Sebastian $\vee$ A, Leib Stephen L, Sturzenegger Mathias SP. Enzephalitis beim Erwachsenen. Swiss Med Forum. 2017;17:492-8. https://doi.org/10.4414/ smf.2017.02972.

10. Kaiser R. The clinical and epidemiological profile of tick-borne encephalitis in southern Germany 1994-98: a prospective study of 656 patients. Brain. 1999;122(Pt 1):2067-78 http://www.ncbi.nlm.nih.gov/pubmed/ 10545392.

11. Mickiene A, Laiskonis $A$, Günther G, Vene S, Lundkvist A, Lindquist L. Tickborne encephalitis in an area of high endemicity in Lithuania: disease severity and long-term prognosis. Clin Infect Dis. 2002;35:650-8. https:// doi.org/10.1086/342059.

12. Thakur KT, Motta M, Asemota AO, Kirsch HL, Benavides DR, Schneider EB, et al. Predictors of outcome in acute encephalitis. Neurology. 2013;81:793-800. https://doi.org/10.1212/WNL.0b013e3182a2cc6d.

13. Mailles A, De Broucker T, Costanzo P, Martinez-Almoyna L, Vaillant V, Stahl $J-P$, et al. Long-term outcome of patients presenting with acute infectious encephalitis of various causes in France. Clin Infect Dis. 2012;54:1455-64. https://doi.org/10.1093/cid/cis226.

14. Yeshokumar AK, Gordon-Lipkin E, Arenivas A, Cohen J, Venkatesan A, Saylor D, et al. Neurobehavioral outcomes in autoimmune encephalitis. J Neuroimmunol. 2017;312:8-14. https://doi.org/10.1016/j.jneuroim.2017. 08.010 .

15. Karelis G, Bormane A, Logina I, Lucenko I, Suna N, Krumina A, et al. Tickborne encephalitis in Latvia 1973-2009: epidemiology, clinical features and sequelae. Eur J Neurol. 2012;19:62-8. https://doi.org/10.1111/j.14681331.2011.03434.x.

16. Bogovič P, Stupica D, Rojko T, Lotrič-Furlan S, AvšiččŽupanc T, Kastrin $A$, et al. The long-term outcome of tick-borne encephalitis in Central Europe. Ticks Tick Borne Dis. 2018;9:369-78.

17. Veje M, Nolskog P, Petzold M, Bergström T, Lindén T, Peker Y, et al. Tickborne encephalitis sequelae at long-term follow-up: a self-reported case-control study. Acta Neurol Scand. 2016;134:434-41. https://doi.org/ 10.1111/ane.12561.

18. Veje M, Studahl M, Thunström E, Stentoft E, Nolskog P, Celik Y, et al. Sleep architecture, obstructive sleep apnea and functional outcomes in adults with a history of tick-borne encephalitis. PLoS One. 2021;16:e246767. https://doi.org/10.1371/journal.pone.0246767.

19. Harris PA, Taylor R, Minor BL, Elliott V, Fernandez M, O'Neal L, et al. The REDCap consortium: Building an international community of software platform partners. J Biomed Inform. 2019;95:103208. https://doi.org/10. 1016/J.JBI.2019.103208.

20. Harris PA, Taylor R, Thielke R, Payne J, Gonzalez N, Conde JG. Research electronic data capture (REDCap) — a metadata-driven methodology and workflow process for providing translational research informatics support. J Biomed Inform. 2009;42:377-81. https://doi.org/10.1016/J.JBI.2008. 08.010 .

21. Ellul M, Solomon T. Acute encephalitis - diagnosis and management. Clin Med. 2018;18:155-9. https://doi.org/10.7861/clinmedicine.18-2-155.

22. Taba P, Schmutzhard E, Forsberg P, Lutsar I, Ljøstad U, Mygland Å, et al. EAN consensus review on prevention, diagnosis and management of tick-borne encephalitis. Eur J Neurol. 2017;24:1214-e61. https://doi.org/ 10.1111/ene.13356.

23. European Centre for Disease Prevention and Control. ECDC Meeting Report 2011: Second expert consultation on tick-borne diseases with emphasis on Lyme borreliosis and tick-borne encephalitis. 2012. http:// ecdc.europa.eu/en/publications/_layouts/forms/Publication_DispForm. aspx? List=4f55ad51-4aed-4d32-b960-af70113dbb90\&ID $=584$.

24. Johns MW. A new method for measuring daytime sleepiness: the Epworth sleepiness scale. Sleep. 1991;14:540-5. https://doi.org/10.1093/ sleep/14.6.540.
25. Valko PO, Bassetti CL, Bloch KE, Held U, Baumann CR. Validation of the fatigue severity scale in a Swiss cohort. Sleep. 2008;31:1601-7 http:// www.ncbi.nlm.nih.gov/pubmed/19014080. Accessed 25 Sep 2017.

26. Bastien $\mathrm{CH}$, Vallières $\mathrm{A}$, Morin $\mathrm{CM}$. Validation of the insomnia severity index as an outcome measure for insomnia research. Sleep Med. 2001;2:297-307. https://doi.org/10.1016/S1389-9457(00)00065-4.

27. Kühner $C$, Bürger $C$, Keller F, Hautzinger M. Reliabilität und validität des revidierten Beck- Depressionsinventars (BDI-II). Befunde aus deutschsprachigen stichproben. Nervenarzt. 2007;78:651-6. https://doi.org/10.1007/ s00115-006-2098-7.

28. Bloch KE, Schoch OD, Zhang JN, Russi EW. German version of the Epworth sleepiness scale. Respiration. 1999;66:440-7. https://doi.org/10. $1159 / 000029408$

29. Gavrilov YV, Alekseeva TM, Kreis OA, Valko PO, Weber KP, Valko Y. Depression in myasthenia gravis: a heterogeneous and intriguing entity. J Neurol. 2020;267:1802-11. https://doi.org/10.1007/s00415-020-09767-7.

30. Gerber M, Lang C, Lemola S, Colledge F, Kalak N, Holsboer-Trachsler E, et al. Validation of the German version of the insomnia severity index in adolescents, young adults and adult workers: results from three crosssectional studies. BMC Psychiatry. 2016;16:174. https://doi.org/10.1186/ s12888-016-0876-8.

31. Bargiotas P, Dietmann A, Haynes AG, Kallweit U, Calle MG, Schmidt M, et al. The Swiss narcolepsy scale (SNS) and its short form (sSNS) for the discrimination of narcolepsy in patients with hypersomnolence: a cohort study based on the Bern sleep-wake database. J Neurol. 2019;266:213743. https://doi.org/10.1007/s00415-019-09365-2.

32. Allen RP, Picchietti DL, Garcia-Borreguero D, Ondo WG, Walters AS, Winkelman JW, et al. Special section in sleep medicine restless legs syndrome/ Willis-Ekbom disease diagnostic criteria: updated international restless legs syndrome study group (IRLSSG) consensus criteria-history, rationale, description, and significance; 2014. https://doi.org/10.1016/j.sleep.2014. 03.025.

33. De Ory F, Avellón A, Echevarría JE, Sánchez-Seco MP, Trallero G, Cabrerizo $M$, et al. Viral infections of the central nervous system in Spain: a prospective study. J Med Virol. 2013;85:554-62. https://doi.org/10.1002/jmv. 23470.

34. Kupila L, Vuorinen T, Vainionpää R, Hukkanen V, Marttila RJ, Kotilainen P. Etiology of aseptic meningitis and encephalitis in an adult population. Neurology. 2006;66:75-80. https://doi.org/10.1212/01.wnl.0000191407. 81333.00.

35. Venkatesan A, Michael BD, Probasco JC, Geocadin RG, Solomon T. Acute encephalitis in immunocompetent adults. Lancet. 2019;393:702-16. https://doi.org/10.1016/S0140-6736(18)32526-1.

36. Armangue T, Leypoldt F, Dalmau J. Autoimmune encephalitis as differential diagnosis of infectious encephalitis. Curr Opin Neurol. 2014;27:361-8. https://doi.org/10.1097/WCO.00000000000000087.

37. Dubey D, Pittock SJ, Kelly CR, McKeon A, Lopez-Chiriboga AS, Lennon VA, et al. Autoimmune encephalitis epidemiology and a comparison to infectious encephalitis. Ann Neurol. 2018;83:166-77. https://doi.org/10.1002/ ana.25131.

38. Baroutsou V, Zens KD, Sinniger P, Fehr J, Lang P. Analysis of tick-borne encephalitis vaccination coverage and compliance in adults in Switzerland, 2018. Vaccine. 2020:38:7825-33. https://doi.org/10.1016/j.vaccine. 2020.10.022.

39. Guo $Y$, Tian $X$, Wang $X$, Xiao Z. Adverse effects of immunoglobulin therapy. Front Immunol. 2018;9:1299. https://doi.org/10.3389/fimmu. 2018.01299.

40. Brown JR, Bharucha T, Breuer J. Encephalitis diagnosis using metagenomics: application of next generation sequencing for undiagnosed cases. J Inf Secur. 2018;76:225-40. https://doi.org/10.1016/j.jinf.2017.12.014.

41. Wilson MR, Sample HA, Zorn KC, Arevalo S, Yu G, Neuhaus J, et al. Clinical metagenomic sequencing for diagnosis of meningitis and encephalitis. N Engl J Med. 2019;380:2327-40. https://doi.org/10.1056/NEJMoa1803396.

42. Hotopf M, Noah N, Wessely S. Chronic fatigue and minor psychiatric morbidity after viral meningitis: a controlled study. J Neurol Neurosurg Psychiatry. 1996;60:504-9. https://doi.org/10.1136/jnnp.60.5.504.

43. Schmidt H, Cohrs S, Heinemann T, Goerdt C, Djukic M, Heimann B, et al. Sleep disorders are long-term sequelae of both bacterial and viral meningitis. J Neurol Neurosurg Psychiatry. 2006;77:554-8. https://doi.org/10. 1136/jnnp.2005.071142. 
44. Schmidt H, Heimann B, Djukic M, Mazurek C, Fels C, Wallesch C-W, et al. Neuropsychological sequelae of bacterial and viral meningitis. Brain. 2006;129:333-45. https://doi.org/10.1093/brain/awh711.

45. Tubiana S, Varon E, Biron C, Ploy M-C, Mourvillier B, Taha M-K, et al.

Community-acquired bacterial meningitis in adults: in-hospital prognosis, long-term disability and determinants of outcome in a multicentre prospective cohort. Clin Microbiol Infect. 2020;26:1192-200. https://doi. org/10.1016/j.cmi.2019.12.020.

\section{Publisher's Note}

Springer Nature remains neutral with regard to jurisdictional claims in published maps and institutional affiliations.

- fast, convenient online submission

- thorough peer review by experienced researchers in your field

- rapid publication on acceptance

- support for research data, including large and complex data types

- gold Open Access which fosters wider collaboration and increased citations

- maximum visibility for your research: over 100M website views per year

At BMC, research is always in progress.

Learn more biomedcentral.com/submissions 